\title{
Mixed effects and mechanisms of cannabinoids for triple-negative breast cancer treatment
}

\author{
Khanh Tran (MD, PhD) \\ Department of Molecular Medicine and Pathology \\ University of Auckland, Auckland, New Zealand \\ Correspondence: Dr Khanh Tran. Email: kb.tran@auckland.ac.nz
}

\begin{abstract}
Triple-negative breast cancer (TNBC) is a subtype of breast cancer characterized by the lack of estrogen receptors (ER), progesterone receptors, and HER-2 receptors. Thus, TNBC tumours do not benefit from the current therapies targeting ER or HER-2. Therefore, there is an urgent need to develop novel treatment for this subtype of breast cancer. Marijuana is a common name given to Cannabis plants, a group of plants in the Cannabis genus of the Cannabaceae family. Cannabis plants are among the oldest cultivated crops, traced back at least 12,000 years and are well known for their multi-purpose usage, including medicinal purposes. The main active compounds extracted from Cannabis plants are 21-carbon-containing terpenophenolics, which are referred to as phytocannabinoids. Of these, the tetrahydrocannabinol (THC) group contains highly potent cannabinoids, including delta-9tetrahydrocannabinol $(\Delta 9-\mathrm{THC})$ and delta-8-tetrahydrocannabinol $(\Delta 8-\mathrm{THC})$, which are the most abundant THCs and are largely responsible for psychological and physiological effects of marijuana. The use of Cannabis plants for medicinal purposes was first recorded in $2337 \mathrm{BC}$ in China, where Cannabis plants were used to treat pains, rheumatism, and gout. Recently, several cannabinoids have been approved for a number of treatments, one of which is the treatment of nausea and vomiting caused by chemotherapy in cancer patients. Furthermore, increasing evidence shows that cannabinoids not only attenuate side effects due to cancer treatment, but might also potentially possess direct antitumor effects in several cancer types, including breast cancer. However, anti-tumour activity of marijuana has been variable in different studies and even promoted tumour growth in some cases. In addition, the mechanisms of cannabinoid action in cancer remain unclear. This review summarizes evidence about the mixed actions of cannabinoids in cancer in general and triple-negative breast cancer in particular.
\end{abstract}

Keywords Triple-negative breast cancer, cannabinoid, marijuana, cell signalling, medicinal plants

\section{Introduction}

Breast cancer is the most commonly diagnosed cancer in women worldwide. In 2019, the total number of female patients diagnosed with breast cancer for the first time worldwide was approximately 2 million, which accounted for $18.9 \%$ of all female cancer incidence [1]. The incidence of breast cancer in developed countries is 66.4/100,000 people, which is twice as high as that in developing countries [2]. In New Zealand, the rate of breast cancer in 2019 was approximately 80 per 100,000 [1]. Geographical regions that have high incidences of breast cancer include West and North Europe, Australia/New Zealand and North America [1]. It has been estimated that the possibility for a woman to develop breast cancer in her lifetime is approximately one in ten [3]. Although mammography screening has decreased the breast cancer death rates $[4,5]$, breast cancer remains the leading cause of cancer mortality in women. 
In 2019, there were approximately 689,000 breast cancer deaths for females globally, accounting for $15.9 \%$ of total female cancer mortality [1]. Breast cancer is a burden for patients and society and like with any other malignant diseases, being diagnosed with breast cancer is always an acute emotional shock, which may permanently and substantially affect the physical and intellectual capacity of the patients $[6,7]$. In Sweden. the total annual cost required from a patient younger than 50 years of age with metastatic breast cancer was estimated to $\$ 43,565$ USD [8]. In France, trastuzumab accounted for $44 \%$ of total treatment cost (Poncet, 2009) and in Canada, annual cost for trastuzumab required for a metastatic breast cancer patient was $\$ 28,350$ USD [9]. Therefore, it is imperative that breast cancer be effectively managed, and for that purpose, searching for novel treatment is crucial.

\section{Classification of breast cancer}

It has been established that the expression status of estrogen receptor (ER), progesterone receptor (PR) and HER-2 imposes significant influences on the development, prognosis and treatment outcome of breast cancer [10]. Therefore, breast cancer has been classified according to differential expression of ER, PR and HER-2. Based on the presence of ER in cancer cells, breast cancer is classified into ER positive $(\mathrm{ER}+)$, which expresses the estrogen receptor $\alpha$ gene, and ER negative, which lacks the estrogen $\alpha$ gene. The survival and growth of ER + breast cancer cells are dependent on estrogen binding, whereas ER- cells rely on other growth factors, including epidermal growth factor (EGF) and endothelial vascular growth factor [11]. ERbreast cancer accounts for approximately $75 \%$ of all breast cancer. Compared to ER+, ERbreast cancer is often associated with a poorer prognosis and more aggressive progression. In contrast to ER+, ER- is unlikely to respond to hormonal treatments [12]. The presence of PR in the cancer cells, along with ER, is more predictive of a hormonal responsive tumor than the presence of ER alone $[13,14]$. Tumors with $\mathrm{ER}+/ \mathrm{PR}+$ were reported to exhibit a response rate of $75 \%$ to hormonal therapies, compared to the response rate of $33 \%$ in ER+/PR- tumors [15]. Furthermore, approximately one in five breast cancer patients exhibit amplification of the HER-2 gene and overexpression of the receptor. HER-2 positive breast cancer is associated with more aggressive behaviors of the tumors [16]. In addition, HER-2 positive breast cancer is also unlikely to respond to hormonal therapies. However, they respond to anti-HER-2 monoclonal antibody such as trastuzumab, a specific targeted treatment for HER-2 positive breast cancer [12].

\section{Triple-negative breast cancer}

Triple negative breast cancer (TNBC) is a subtype of breast cancer that is characterized by the lack of ER, PR and HER-2. TNBC accounts for 10-20\% of all breast cancer [11]. Compared to other subtypes of breast cancer, clinical features of TNBC include poor outcome, aggressive progression of the primary tumors and metastasis, shorter survival and high mortality rate. In a cohort study of 1601 breast cancer patients, Dent (2007) found that $11.2 \%$ of the patients were of TNBC subtype [17]. In addition, the results showed that TNBC has an increased risk of distant recurrence and death within 5 years, but not thereafter. Similarly, it has been noted that there is a sharp decrease in survival during the first 5 years after diagnosis of TNBC. A study on 496 patients with invasive breast cancer from the Carolina Breast Cancer Study showed that TNBC accounted for $26 \%$ of the total breast cancer patients in the study [18]. TNBC tumors were mainly grade 3 with high mitotic index. In addition, TNBC was more frequent in premenopausal patients and often associated with overexpression of EGFR and p53 [19]. TNBC was also reported to closely relate to BCRA1 mutation. It was reported that $75 \%$ of breast cancer tumors with BCRA1 mutation have a TNBC phenotype [10]. TNBC lacks ER and HER-2, therefore TNBC do not benefit from endocrine therapy or trastuzumab [12]. The major treatment options for TNBC are surgery, chemotherapy and radiation. In addition to 
these conventional treatment options, several targeted therapies including EGFR inhibitors, PARP inhibitors and angiogenesis inhibitors are in clinical trials [20,21]. As there are currently no targeted treatment for TNBC, there is an urgent need to develop novel treatment for this subtype of cancer.

\section{Marijuanna-derived compounds}

Cannabinoids can be classified into three main groups, including natural cannabinoids that are derived from the plant Cannabis sativa (phytocannabinoids), endogenous cannabinoids that are originated within the body (endocannabinoids), and cannabinoid analogues that are artificially synthesied (synthetic cannabinoids) [22, 23]. Phytocannabinoids are 21-carboncontaining terpenophenolic compounds derived from the plants Cannabis sativa and Cannabis indica. At least 85 phytocannabinoids has been isolated and chemically characterized. Based on their chemical structures, phytocannabinoids are further categorized into different groups. Of these, the tetrahydrocannabinol (THC) group contains highly potent cannabinoids, including delta-9-tetrahydrocannabinol $(\Delta 9$-THC) and delta-8-tetrahydrocannabinol $(\Delta 8$ THC), which are the most abundant and largely responsible for psychological and physiological effects of marijuana [23]. After the presence of CB1 and CB2 cannabinoid receptors in the brain and the immune system was recognized, another group of cannabinoids has been found. Endocannabinoids are a group of ligands for cannabinoid receptors that are endogenously biosynthesized within the body. Endocannabinoids are all arachidonic acid derivatives, including arachidonoylethanolamine (anandamide or AEA), 2- arachidonoyl glycerol (2-AG), 2-arachidonoyl glyceryl ether (noladin ether), N-arachidonoyl-dopamine (Gupta GP), and $O$-arachidonoyl-ethanolamine (Virodhamine or OAE) [24, 25]. Endocannabinoids and cannabinoids receptors present within the body are two major components of the endocannabinoid system, which has now been revealed to have important roles in modulation of neurotransmitter release; control of cell survival, transformation, proliferation and metabolism; regulation of pain perception, cardiovascular, gastrointestinal and respiratory functions [26].

Synthetic cannabinoids are a large group of structurally diverse compounds that are able to bind to the cannabinoid receptors and have cannabimimetic activities. Classical synthetic cannabinoids refer to compounds that retain parts of the dibenzopyran ring of THC [27]. The first generation of classical synthetic cannabinoids includes HU-210 and Nabilone (Cesamet ${ }^{\circledR}$, Lilly) the latter of which was approved by the U.S Food and Drug Administration (FDA) for treatment of chemotherapy-induced nausea and vomiting [28]. The second generation of classical synthetic cannabinoids synthesized by JW Huffman's group has a variety of core ring structures. The compounds of this group are named as JWH- compounds to honor JW Huffman, the creator of JWH-018 and other JWH- compounds, including JWH-133, JWH-018, JWH075 and JWH-019 [29]. In contrast to classical synthetic cannabinoids, non-classical compounds lack the dibenzopyran in their molecular structures, including cyclohexylphenol compounds and aminoalkylindole (AAI) compounds. CP cannabinoids were developed by Pfizer and include CP-47,497 and CP-55,940 [30]. Aminoalkylindole (AAI) cannabinoids were developed at Sterling Winthrop, with WIN55,212-2 to be the most potent compound in this series [31].

\section{Cannabinoid receptors}

To date, two specific receptors for cannabinoid ligands have been cloned from mammalian cells. The first cannabinoid receptor, the CB1 receptor, was cloned in 1990 from a rat cerebral cortex cDNA library [32]. The second cannabinoid receptor, CB2 receptor, was cloned in 1993 by Munro et al. from human promyelocytic leukemic HL-60 cells [33]. Although the two receptors have a large number of common cannabinoid ligands, they differ substantially from 
each other in many aspects, including amino acid sequence, distribution location within the body and downstream signaling cascades. Human and rat CB1 receptor proteins consist of 427 and 473 amino acids, respectively, with $97-99 \%$ amino acid sequence identity across species.

CB2 receptor was cloned from human leukemic HL-60 cells as a cDNA fragment that encodes a protein of 360 amino acids, with $82 \%$ and $81 \%$ amino acid sequence identity to mouse and rat $\mathrm{CB} 2$ receptors, respectively. $\mathrm{CB} 1$ and $\mathrm{CB} 2$ receptor proteins show $44 \%$ identity in general and $68 \%$ for the transmembrane residues, which are considered to determine ligand specificity for the receptors [23]. The distribution location of CB1 and CB2 cannabinoid receptors is also different. CB1 receptors are found primarily in the brain and are the most abundant G proteincoupled receptor in the brain. Particularly, the CB1 receptor is highly expressed in basal ganglia and cerebellum, cortex and hippocampus, amyglada, thalamus, hypothalamus, pons, and medulla [23]. In addition, the CB1 receptor is also expressed in peripheral nerve terminals and extraneural tissues such as testes, eyes, vascular endothelium and spleen [34]. CB2 receptors are found primarily in the immune cells (B and T cells and macrophages) and tissues (spleen, tonsils and lymph nodes) [35].

Cannabinoid receptors belong to the super family of G protein-coupled receptors (GPCRs), also known as seven-transmembrane domain (7TM) receptors [36]. It has been established that CB1 receptors are coupled to $G_{i / o}$ and $G_{s}$ proteins, while CB2 receptors are coupled to $G_{i / o}$ proteins $[37,38]$. Activation of $\mathrm{CB} 1$ and $\mathrm{CB} 2$ receptors leads to inhibition of adenylyl cyclase. In addition, activated cannabinoid receptors can also modulate signaling cascades that are involved in regulation of cell survival, growth, and proliferation [39]. Major downstream signaling cascades of cannabinoid receptors include extracellular signal-regulated kinase, cJun N-terminal kinase and p38 mitogen-activated protein kinase, phosphatidylinositol 3kinase/Akt and focal adhesion kinase [40]. In addition, CB1 receptor can modulate certain types of ion channels, which plays a crucial role in neuromodulatory actions of the endocannabinoids. CB1 receptor can inhibit N-, L- and P- or Q-type voltage-sensitive $\mathrm{Ca}^{2+}$ channels [41, 42] and activate $\mathrm{G}$ protein-activated inwardly rectifying $\mathrm{K}^{+}$channels [43].

\section{Anticancer effects of cannabinoids in triple-negative breast cancer}

TNBC lacks ER and HER2, the targets for selective estrogen receptor modulators (SERMs) and anti-HER2 monoclonal antibodies. Therefore, it is imperative that alternative treatment for TNBC be developed in order to effectively manage this poor prognosis and highly aggressive subtype of breast cancer. Due to the lack of specific treatment for TNBC and to the fact that cannabinoids produced antitumoral effects in some other cancers, recent attention has also been drawn to the possibility for cannabinoids to be used as a treatment for TNBC [44].

It has been reported that cannabinoid receptors are overexpressed in primary human breast tumors compared to normal breast tissue [44]. In breast cancer tissue, Caffarel (2006) showed that CB2 expression was higher than CB1 expression in the same tumor [45]. In addition, CB2 expression appeared to correlate positively with the grades of the tumors. Compared to $\operatorname{ER}(+)$, $\mathrm{PR}(+)$, and HER2 $(+)$ breast cancer tumors, CB2 mRNA were found to be higher than in ER(), PR(-), and HER2(-), respectively [45]. Studies have also confirmed the presence of CB1 and CB2 receptors in TNBC cells using reverse transcriptase and real-time PCR coupled with confocal microscopy $[44,46]$.

In vitro, treatment with cannabinoids has been carried out in various TNBC cells, of which the most commonly used cell lines are MDA-MB-231 and MDA-MB-468. Studies demonstrated that cannabinoids inhibit the survival and proliferation of TNBC cells in a dosedependent and time-dependent manner [45]. In addition, using CB1 and CB2 receptor antagonists was found to prevent cannabinoid-induced cell death, suggesting that the inhibitory 
effects of cannabinoids against TNBC cells were mediated via cannabinoid receptors. Antiproliferative effects of cannabinoids in vitro can be attributed to induction of apoptosis and cell cycle arrest [44, 47].

In vivo, cannabinoids have been reported to suppress the growth of TNBC xenografts. In a study conducted by Ligresti et al. (2006), MDA-MB-231 cells were subcutaneously inoculated into the dorsal right side of male athymic mice [47]. The mice were intratumorally treated with $\Delta 9$-THC or cannabidiol $(5 \mathrm{mg} / \mathrm{kg})$ twice a week for 16 days. It was found that $\triangle 9-T H C$ and cannabidiol significantly reduced the volume of the xenografts. Using SCID mice, Qamri (2009) reported that both JWH-133 and WIN55,212-2 suppressed the growth of MDA-MB231 xenografts, and the effects were mediated via CB1 and CB2 receptors. Cannabinoids have been also reported to inhibit the metastasis of TNBC tumors [44]. Treatment with cannabidiol (i.p. injection of $5 \mathrm{mg} / \mathrm{kg}$ every $72 \mathrm{~h}$ for 21 days) significantly reduced metastatic lung infiltration from the primary tumors induced by injection of MDA-MB-231 cells into the left paw of the mice [47]. Similarly, treatment with JWH-133 and WIN55,212-2 was shown to reduce lung metastasis by $65 \%$ to $80 \%$ respectively [44].

The involvement of $\mathrm{CB} 1$ and $\mathrm{CB} 2$ receptors has been reported both in vitro and in vivo. $\mathrm{CB} 1$ and $\mathrm{CB} 2$ receptor antagonists have been demonstrated to reverse the inhibitory effects of cannabinoids on cultured TNBC cells and on TNBC xenografts, suggesting cannabinoidinduced cell death in vitro and tumor growth suppression in vivo were mediated via $\mathrm{CB} 1$ and $\mathrm{CB} 2$ receptors. Furthermore, the mediative role of $\mathrm{CB} 2$ receptor has been confirmed using $\mathrm{CB} 2$ targeting siRNA that was transfected into MDA-MB-231 cells. The results showed that CB2 siRNA was able to block JWH-133 and WIN55,212-2 effects by decreasing level of CB2 expression in the transfected [44]. Taken together, studies indicate that the anticancer effects of cannabinoids in TNBC were mediated via $\mathrm{CB} 1$ and $\mathrm{CB} 2$ receptor.

\section{Cellular mechanisms of anticancer effects of cannabinoids}

So far, several mechanisms for anticancer effects of cannabinoids have been identified, including apoptosis induction, cell cycle arrest, antiangiogenesis and inhibition of migration and invasion. Cannabinoids were found to induce apoptosis in glioma cells and other cancer cells in culture [48-50]. Cannabinoids also increased apoptotic activities in tumors treated with cannabinoids, which was associated with the suppression of the tumor growth $[51,52]$. Existing evidence indicated that cannabinoid-induced apoptosis was mediated via the activation of cannabinoid receptors, which in turn triggers the proapoptotic mitochondrial intrinsic pathway. In glioma cells and pancreatic cancer cells, activation of cannabinoid receptors resulted in two peaks of ceramide generation by the mechanisms of sphingomyelin hydrolysis and de novo synthesis, respectively $[53,54]$. Studies showed that the second peak of ceramide accumulation accounted for the apoptosis action induced by cannabinoids [55]. The mechanism by which accumulation of the spingolipid ceramide leads to apoptosis has been reported to be mediated by the stress-regulated protein $\mathrm{p} 8$, which is upregulated by ceramide accumulation. $\mathrm{p} 8$ upregulation leads to the upregulation of the activating transcription factor 4 (ATF-4) and the $\mathrm{C} / \mathrm{EBP}-$ homologous protein (CHOP) and through which induce apoptosis [56].

Cannabinoids also cause cell cycle arrest in cancer cells of prostate carcinoma [56], thyroid epithelioma [57], breast carcinoma [44], lung carcinoma [51], and gastric carcinoma [58]. It has been suggested that activation of cannabinoid receptors lead to the inhibition of adenylyl cyclase and the cAMP/protein kinase A (PKA) pathway. As PKA inhibits Raf-1, cannabinoids prevent the inhibition of Raf-s and consequently result in prolonged activation of Raf1/MEK/ERK signaling cascade. The activation of Raf-1/MEK/ERK has been found to be associated with cell arrest in various cancer cells. Also, prolonged activation of Raf- 
1/MEK/ERK may induce cell cycle arrest by modulating the expression of molecules that involve in the cell cycle regulation, including $\mathrm{p} 16^{\text {Ink4a }}, \mathrm{p} 15^{\text {Ink4b }}$ and $\mathrm{p} 21^{\text {Cip } 1}$, which can lead to the cell cycle arrest at the $\mathrm{G} 1$ phase $[59,60]$. In addition, cannabinoid-induced cell cycle arrest in thyroid epithelioma was also reported to be mediated via the induction of the cyclindependent kinase inhibitor $\mathrm{p} 27^{\mathrm{kip} 1}[61]$.

For a tumor to grow beyond the minimal size, it must recruit new blood vessels by producing proangiogenic factors that promote the formation of new vessels for nutrition, gas exchange, and waste disposal. Targeting the formation of new blood vessels in the tumor, therefore, is an important approach in anticancer drug development. Studies indicated that cannabinoid treatment can change the blood vessel pattern in xenograft tumors from a hyperplasic network of dilated vessels to a pattern of blood vessels characterized by narrow, differentiated and impermeable capillaries [62]. The anti-angiogenesis effects of cannabinoids is associated with inhibition of the expression of VEGF and other proangiogenic factors such as placental growth factor and angiopoietin 2 [61]. In addition, cannabinoids can also inhibit the formation of new blood vessels by downregulating the expression of VEGF receptors [61]. It has been suggested that ceramide synthesis de novo involved in the mechanism of antiangiogenesis of cannabinoids. Inhibition of ceramide synthesis de novo prevented the cannabinoid-induced inhibition of VEGF production in vitro and in vivo [39].

Recently, increasing evidence shows that cannabinoids can also inhibit the invasiveness of cancer tumors. WIN55,212-2 and JWH-015 significantly decreased in vitro chemotaxis and chemoinvasion of lung cancer cells and inhibited in vivo metastasis from the xenografts to the lungs [51]. Similarly, 2-methyl-arachidonyl-2'-fluoro-ethylamide (Met-F-AEA) significantly reduced the number of metastatic nodes following injection of Lewis lung cancer cells into the paw [61]. Mechanisms for the anti-migration and anti-invasion effects of cannabinoids may be related to the inhibition of Akt, which is involved in the regulation of migration. In addition, cannabinoids were also found to downregulate the expression and activity of matrix metalloproteinase-2 (MMP2), which plays an important role in tissue remodeling and closely associated with angiogenesis, tissue repair and metastasis [44, 62].

\section{Molecular mechanisms of cannabinoids}

\section{Modulation of Receptor Expression by Synthetic Cannabinoids}

In normal noncancerous human breast tissue, cannabinoid receptors are expressed at lower levels compared to breast cancer tissue [44]. In breast cancer tissue, it was reported that CB2 expression was higher than the $\mathrm{CB} 1$ expression in the same tumors and CB2 expression seemed to correlate with the grades of the tumors [45]. Compared to $\mathrm{ER}(+)$ and $\mathrm{PR}(+)$ breast cancer tumors, CB2 mRNA were found to be 3.6- and 2.3-fold in ER(-) and PR(-) tumors, respectively [45].

Alteration in cannabinoid receptor expression was also associated with cannabinoid exposure. Chronic treatment with cannabinoids led to downregulation of cannabinoid receptors [63-65]. Daily treatment with $\triangle 9$-THC for 14 days caused a $30 \%$ reduction in cannabinoid receptor binding [66]. Downregulation of cannabinoid receptors following long-term treatment with cannabinoid is attributed to the internalization and degradation of the receptors. Cannabinoid receptors belong to G protein-coupled receptors (GPCRs); and like many other GPCRs, cannabinoid receptors undergo agonist-induced or constitutive internalization from the cell membrane to low $\mathrm{pH}$ endosomes, where a certain amount of the receptors is recycled back to the cell surface, another part stays in the cytoplasm and contributes to the intracellular pool of 
the receptors, and the rest of the endocytosed receptors are sent to lysosomes for degradation $[67,68]$.

The intracellular reservoir of CB1 receptor is reported to account for $85 \%$ of total cellular CB1 cannabinoid receptor, however functions of the intracellular pool remain to be elucidated. As reviewed by Rozenfeld (2011), the intracellular pool of cannabinoid receptors serves as a source from which surface CB1 receptors are replenished for endocytosed receptors [69]. However, Grimsey (2010) reported that the intracellular pool of the cannabinoid receptors does not contribute to the recycling to the cell surface receptor population [70]. Mechanisms for cannabinoid receptor internalization and recycle are not fully established. HU308-induced endocytosis of CB2 cannabinoid receptors was found to be mediated via Rab5, a small GTPase localised to early endosomes, while $\mathrm{CB} 2$ recycle was mediated via a recycling endosomeRab11-dependent pathway [71]. WIN55,212-2 induced CB1 and CB2 downregulation and CB1 receptors were sorted in lysosomal compartments for degradation by a G-proteinassociated sorting protein (GASP-1) and an adaptor protein 3 (AP-3) [65, 72].

In contrast to $\mathrm{ER}(+)$ breast cancer where estrogen-ER-ERE pathway plays the central role in cellular processes, ER(-) breast cancer is under the regulation from other signalling pathways that are independent of ER. Previous findings have demonstrated that overexpression of EGFR is common in ER(-) breast cancer [73], suggesting EGFR signalling may play important roles in regulating the cancer cell fate. Dominant negative EGFR tumors presents a strong reduction of vascular epidermal growth factor (VEGF) expression and an increase in the apoptotic rate. And EGFR-dependent Ha-ras activation has a crucial role in VEGF expression, tumor angiogenesis and growth [74]. The vital importance of EGFR in various cancer types has made it a key target for cancer treatment therapies, with the general goal being EGFR downregulation. Downregulation in EGFR expression after cannabinoid treatment has been previously reported in various cancer types. Anandamide induced cell death and a decrease of EGFR levels on LNCaP, DU145 and PC3 prostate cancer cells and inhibited the EGFstimulated growth of the cells (Mimeault, 2003). Another cannabinoid, WIN55,212-2, was reported to induce growth inhibition of PDV.C57 epidermal tumor xenografts and decrease EGFR and phosphorylated EGFR [62].

\section{Modulation of Downstream Signaling of EGFR and Cannabinoid Receptors}

An increasing number of studies have demonstrated that p38-MAPK plays a crucial role in cannabinoid receptor downstream signalling, in which activation of p38-MAPK is associated with apoptosis induction. Cannabinoid-induced activation of p38 MAPK has been reported in vitro cell lines and in a number of other cancer types. Also, p38-MAPK-related apoptosis following cannabinoid treatment in various cancer cell types. In Jurkat human leukemia cells, $\triangle 9$-THC and JWH-133 treatment induced CB2-mediated cell death and activation of p38MAPK (Herrera, 2005). In mantle cell lymphoma (MCL) cells, R(+)-methanandamide and WIN55,212-2 were reported to induce apoptosis via a sequence of events, including accumulation of de novo synthesized ceramide, activation of p38 MAPK and depolarization of the mitochondrial membrane [75]. Not only in cancer cells, cannabinoid-induced activation of p38 MAPK also occurs in central nervous system cells, however the effect is mediated via CB1 cannabinoid receptor. In rat and mouse hippocampal slices, anadamide, 2arachidonoylglycerol, WIN55,212-2 and $\Delta$ 9-THC activated $\mathrm{p} 38$ MAPK via CB1 receptor, but the cannabinoids did not activate c-Jun N-terminal kinase (JNK), another mitogen-activated protein kinase [76]. 
The mechanisms on how activation of cannabinoid receptors leads to activation of p38 MAPK is still not fully understood. In some cell types, G protein-coupled receptors can stimulate p38 MAPK and JNK activities via the protein kinase $\mathrm{C}$ and the tyrosine kinase Src [76]. In other cells from hippocampus, using a specific inhibitor of the Src-family kinase, PP2, did not prevent the cannabinoid-induced activation of $\mathrm{p} 38$ MAPK, suggesting that activation of $\mathrm{p} 38$ MAPK by cannabinoids is independent of Src-family kinases in these cells [76]. Another mechanism for p38 MAPK activation is that CB1 activation stimulates PI3K [77], which in turn can be upstream of p38 MAPK in some certain cell types [78]. After being activated, the following targets of p38 MAPK are of Bcl-2 family proteins. Specifically, Cai (2006) reported that apoptosis of PC12 pheochromocytoma cells by sodium arsenite treatment may be due to direct phosphorylation of a Bcl-2 family protein, Bim, at Ser-65 by p38 MAPK [79]. Alternatively, p38 MAPK can also be upstream of caspase activation [80].

It is commonly implicated that ERK activation leads to cell proliferation. However, increasing evidence indicated that biological effects of ERK activation depend on several factors, of which the duration of ERK activation acts as a key factor. Prolonged activation of ERK induces cell death via apoptosis rather than proliferation [40, 81]. Mechanisms for cannabinoid-stimulated activation of ERK include prolonged accumulation of ceramide and/or inhibition of the adenylyl cyclase (AC)- protein kinase A (PKA) pathway. Activation of cannabinoid receptors led to two peaks of ceramide generation; the short-term peak is associated with sphingomyelin hydrolysis via sphingomyelinase, while the long-term is associated with palmitoyltransferase induction and enhanced ceramide synthesis de novo $[77,82]$. The second peak of ceramide accumulation is related to ERK activation and apoptosis through a mechanism, in which ceramide directly binds to the ceramide-binding motif of Raf- 1 and results in Raf-1 activation. Another mechanism is that cannabinoids inhibit the AC/PKA pathway, through which inhibit indirectly Raf-1. By preventing inhibitory effects of Raf-1, cannabinoids can indirectly activate MEK/ERK and consequently induce apoptosis [83]. Taken together, increasing evidence suggest that activation of MAPKs pathways is likely to play a crucial role for the tumor suppression effect of cannabinoids.

$\mathrm{NF}-\kappa \mathrm{B}$ is a key regulator for the genes involving in immune responses and anti-apoptotic activities [84]. Importantly, NK- $\kappa \mathrm{B}$ belongs to downstream signaling pathways of both cannabinoid receptors and EGFR. Activated NF- $\kappa B$ is found in many cancer types, and studies have showed that activation of NF- $\mathrm{KB}$ can initiate the expression of genes encoding antiapoptotic, angiogenesis, cell cycle regulatory and growth factors, which promote the formation and development of malignant tumors. Conversely, inhibition of NF- $\kappa B$ results in increased apoptotic activities and cell cycle arrest, consequently inducing tumor regression. Therefore, $\mathrm{NF}-\kappa \mathrm{B}$ is an important target for cancer treatment therapies. NF- $\kappa \mathrm{B}$ inhibition by cannabinoids and other compounds has been reported in a number of in vitro studies and with other cancer types. The inhibition of NF- $\mathrm{kB}$ in the tumors may be partly responsible for the tumor regression observed in mice treated with WIN55,212-2. Similarly, AS602868, a specific inhibitor of IKK2, blocked NF- $\kappa \mathrm{B}$ activation and led to apoptosis in human primary acute myeloid leukemia (AML) cells [85]. Non-specific inhibitors of NF- $\kappa$ B such as anti-inflammatory agents and non-steroidal anti-inflammatory drugs (NSAIDs) mediate the regression of adenomatous polyps of the colon, prevent the development of colon cancer, and increase cancer cell apoptosis in existing malignant tumors [86].

In A549 lung adenocarcinoma epithelial cells, anadamide was found to inhibit TNF $\alpha$-induced NF- $\kappa B$ activation by direct inhibition of the I $\kappa B$ kinase (IKK) $\beta$ and the IKK $\alpha$ subunits of $\kappa B$ inhibitor (IאB) kinase complex [87]. To determine the involvement of cannabinoid receptors, 
Sancho (2003) utilized A549 and 5.1 cell lines of expressing only CB1 and CB2 cannabinoid receptors, respectively. The results showed that anandamide-induced NF- $\kappa B$ inhibition was independent of both $\mathrm{CB} 1$ and CB2 cannabinoid receptors. WIN,55212-induced downregulation of NF- $\kappa \mathrm{B}$ expression suggests a potential of the compound to be used as an adjuvant treatment as NF- $\kappa \mathrm{B}$ activation may induce the expression of the multidrug resistance P-glycoprotein, and inhibition of NF- $\kappa \mathrm{B}$ has shown to increase the apoptotic response to chemotherapy and radiation therapy [88]. Also, mere inhibition of NF- $\kappa$ B may be insufficient for a pronounced apoptotic response, therefore combinations of NF- $\kappa B$ inhibitors and conventional chemo/radio therapies may enhance the effectiveness of the treatment and reduce the risk for drug resistance. WIN55,212-2 inhibited the expression of NF- $\kappa \mathrm{B}$ in vivo, therefore it would be interesting to further evaluate WIN55,212-2 as a potential adjuvant treatment for TNBC by combining WIN55,212-2 treatment in conjunction with cytotoxic agents such as anthracyclins or platinum compounds, and/or with radiation therapy to determine whether cotreatment with WIN55,212-2 can increase sensitivity of TNBC cancer cells to chemo/radio therapies, thereby enabling a decreased dose.

Beside the MAPKs pathways, the survival PI3K/Akt/mTOR pathway is also involved in downstream signaling of both cannabinoid receptors and EGFR. It has been well established that the PI3K/Akt/mTOR pathway plays a pivotal role in cellular survival processes, including cell growth, proliferation, invasion and migration. Common results from previous studies indicated that the inhibitory effects of cannabinoids in cancer cells were frequently associated with Akt inhibition, and conversely sustained activation of Akt were related to the protective effects of cannabinoids in neuronal cells. Therefore, PI3K/Akt/mTOR pathway is considered an important target for novel treatments. Cannabinoid-induced inhibition of Akt was found to result from de novo synthesis of ceramide. In glioma cells, cannabinoids-induce intracellular ceramide accumulation by mechanisms of sphingomyelin hydrolysis and ceramide synthesis de novo $[54,89]$. In turn, de novo synthesized ceramide leads to activation of ERK and inhibition of Akt. Blockade of the synthesis de novo of ceramide by L-cycloserine prevented THC-induced ERK activation and THC-induced Akt inhibition [77]. In addition to Akt inhibition, Akt activation by cannabinoid treatment was also reported. Lung carcinoma and glioblastoma cells treated with either THC, WIN55,212-2 or HU-210 showed activation of both ERK and Akt. The activating effect was abolished by blockade of EGFR signal transactivation with the selective EGFR inhibitor AG1478 or with the metalloprotease inhibitor BB94. This suggested the cannabinoid-induced activation of ERK and Akt was dependent on EGFR function [90]. Co-activation of ERK and Akt following cannabinoid treatment was reported to protect astrocytes from ceramide-induced apoptosis [77].

WIN55,212-2 treatment of LNCaP prostate cancer cells resulted in an induction of $\mathrm{p} 27$ and down-regulation of cyclins D1, D2, E [49]. In a study by Portella et al (2003), nude mice inoculated with K-ras-transformed FRTL-5 cells and peritumorally treated with 2-methylarachidonyl-2-fluoro-ethylamide (Met-F-AEA) showed a 50\% reduction in tumor volume compared to vehicle treated mice [61]. Met-F-AEA also resulted in upregulation of p27(kip1) by 1.6-fold, and this upregulation was attenuated by the selective CB1 receptor antagonist, SR141716A, suggesting the cannabinoid-induced upregulation of p27(kip1) was mediated at least in part via $\mathrm{CB} 1$ receptor.

\section{Mixed actions of cannabinoids in other cancer types}

A number of studies also have reported that cannabinoids increased the proliferation of a number of cancer cell types. One possible reason could be that cannabinoids may develop different biological effects on different cell types and their expression levels of cannabinoid 
receptors. Cannabinoids were found to have protective effects towards cultured neurons against excitotoxicity. In contrast, studies also demonstrated cytotoxicity effects of cannabinoids on various cancer cells. Furthermore, even to the same cell type, cannabinoids have also been reported to develop different effects. Delta-9-THC induced hippocampal neuron death through neuronal apoptosis mechanism [91]; the compound was also reported to protect spinal neurons from excitotoxicity produced by kainate [92]. Not only depending on cell types, there exists evidence that cannabinoids may have biological actions in different directions at different ranges of concentrations. Hart et al. reported that THC only induced apoptosis in cancer cells at relatively high concentrations. In contrast, nanomolecular concentrations of THC accelerate the proliferation of the cancer cells in a EGFR- and metalloprotease-dependent mechanism [93]. The difference is important in regard to clinical relevance as after oral or rectal administration of THC or its derivatives, the maximum serum concentrations of THC were only $35-350 \mathrm{nM}[94,95]$.

Evidences suggests that some cannabinoids can trigger the proliferation of cancer cells at suitably low concentrations. In a study by Sanchez (2003), THC and R-/(+)-methanandamide (MET) at nanomolar concentrations induced accelerated proliferation of $\mathrm{PC}-3$ prostate cancer cells. In addition, the stimulation was associated with cannabinoid-induced activation of phosphoinositide kinase-3 (PI3K) cascade and nerve growth factor (NGF) synthesis, a neurotrophic factor previously reported to involve in prostate cells' proliferation (Sanchez, 2003). In other cell types of glioblastoma and lung carcinoma, THC at nanomolar concentrations also accelerated the proliferation of cancer cells and this effect was mediated by EGFR activity in a mechanism where cannabinoid-induced EGFR transactivation was mediated via metalloprotease and tumor necrosis factor $\alpha$-converting enzyme (TACE/ADAM17) [90]. In a study by Sanchez et al., THC and R-/(+)-methanandamide (MET) at nanomolar concentrations induced accelerated proliferation of PC-3 prostate cancer cells. In addition, the stimulation is associated with cannabinoid-induced activation of PI3K cascade and NGF synthesis, a neurotrophic factor previously reported to involve in prostate cells' proliferation [96]. In other cell types of glioblastoma and lung carcinoma, THC at nanomolar concentrations also accelerate the proliferation of cancer cells and this effect was mediated by EGFR activity in a mechanism where cannabinoid-induced EGFR transactivation was mediated via metalloprotease and tumor necrosis factor $\alpha$-converting enzyme (TACE/ADAM17) [93]. JWH133 with the chemical name 3-(1',1'-Dimethylbutyl)-1-deoxy$\triangle 8$-THC possesses a molecular structure that is remarkably similar to that of $\triangle 9$-THC $[97,98]$. It therefore may potentially possess the similar ability to induce accelerated proliferation in cancer cells, which was observed with $\triangle 9$-THC. Another possible mechanism for cannabinoid stimulation effect on tumor growth is that cannabinoids promote the formation of new blood vessels, which better nourish tumor cells and make the tumor to grow faster. It was previously reported that THC induced activation of NGF synthesis [96]; and in a study by Romon, recombinant NGF and NGF produced by breast cancer cells promote breast cancer angiogenesis and endothelial cell invasion [99]. NGF increased the secretion of VEGF in both endothelial and breast cancer cell. NGF also lead to the activation of the PI3K/Akt pathway, which was previously reported to be also activated by THC $[96,100]$. In another aspect, it has been established that EGFR activation may stimulate invasion, angiogenesis and metastasis of cancer tumors [as reviewed by 101]. This may explain why cetuximab, an EGFR monoclonal antibody, is found more effective in vivo, where the drug can develop its effects towards invasion, angiogenesis, and metastasis, than in vitro [102]. THC was found to lead to EGFR transactivation and activation of ERK and Akt/PKB survival pathways in a EGFR-dependent manner [93]. Therefore, further models for assessing the possibility of cannabinoids to promote cancer growth are needed. 


\section{References}

1. Fitzmaurice, C., et al., Global, Regional, and National Cancer Incidence, Mortality, Years of Life Lost, Years Lived With Disability, and Disability-Adjusted Life-Years for 29 Cancer Groups, 1990 to 2017: A Systematic Analysis for the Global Burden of Disease Study. JAMA Oncol, 2019. 5(12): p. 1749-1768.

2. Sung, H., et al., Global Cancer Statistics 2020: GLOBOCAN Estimates of Incidence and Mortality Worldwide for 36 Cancers in 185 Countries. CA: A Cancer Journal for Clinicians, 2021. 71(3): p. 209-249.

3. Jemal, A., et al., Cancer Statistics, 2007. CA: A Cancer Journal for Clinicians, 2007. 57(1): p. 43-66.

4. Coldman, A., et al., Pan-Canadian Study of Mammography Screening and Mortality from Breast Cancer. JNCI: Journal of the National Cancer Institute, 2014. 106(11).

5. Roder, D., et al., Population screening and intensity of screening are associated with reduced breast cancer mortality: evidence of efficacy of mammography screening in Australia. Breast Cancer Research and Treatment, 2008. 108(3): p. 409-416.

6. Giese-Davis, J., et al., Decrease in depression symptoms is associated with longer survival in patients with metastatic breast cancer: a secondary analysis. Journal of clinical oncology : official journal of the American Society of Clinical Oncology, 2011. 29(4): p. 413-420.

7. Mehnert, A. and U. Koch, Prevalence of acute and post-traumatic stress disorder and comorbid mental disorders in breast cancer patients during primary cancer care: a prospective study. Psychooncology, 2007. 16(3): p. 181-8.

8. Lidgren, M., et al., Health related quality of life in different states of breast cancer. Qual Life Res, 2007. 16(6): p. 1073-81.

9. Foster, T.S., et al., The economic burden of metastatic breast cancer: A systematic review of literature from developed countries. Cancer Treatment Reviews, 2011. 37(6): p. 405-415.

10. Foulkes, W.D., I.E. Smith, and J.S. Reis-Filho, Triple-Negative Breast Cancer. New England Journal of Medicine, 2010. 363(20): p. 1938-1948.

11. Cleator, S., W. Heller, and R.C. Coombes, Triple-negative breast cancer: therapeutic options. The Lancet Oncology, 2007. 8(3): p. 235-244.

12. Bauer, K.R., et al., Descriptive analysis of estrogen receptor (ER)-negative, progesterone receptor (PR)-negative, and HER2-negative invasive breast cancer, the so-called triple-negative phenotype. Cancer, 2007. 109(9): p. 1721-1728.

13. Setiawan, V.W., et al., Breast cancer risk factors defined by estrogen and progesterone receptor status: the multiethnic cohort study. American journal of epidemiology, 2009. 169(10): p. 1251-1259.

14. Dowsett, M. and A.K. Dunbier, Emerging biomarkers and new understanding of traditional markers in personalized therapy for breast cancer. Clinical Cancer Research, 2008. 14(24): p. 8019-8026.

15. McGuire, W.L., Steroid receptors in human breast cancer. Cancer research, 1978. 38(11 Part 2): p. 4289-4291.

16. Phillips, G.D.L., et al., Targeting HER2-positive breast cancer with trastuzumab-DM1, an antibody-cytotoxic drug conjugate. Cancer research, 2008. 68(22): p. 9280-9290.

17. Dent, R., et al., Triple-negative breast cancer: clinical features and patterns of recurrence. Clinical cancer research, 2007. 13(15): p. 4429-4434.

18. Carey, L.A., et al., Race, breast cancer subtypes, and survival in the Carolina Breast Cancer Study. Jama, 2006. 295(21): p. 2492-2502. 
19. Rakha, E.A., et al., Prognostic markers in triple-negative breast cancer. Cancer, 2007. 109(1): p. 25-32.

20. Asmis, T., et al., Comorbidity, age and overall survival in cetuximab-treated patients with advanced colorectal cancer (ACRC) — results from NCIC CTG CO. 17: a phase III trial of cetuximab versus best supportive care. Annals of oncology, 2011. 22(1): p. 118-126.

21. Gerber, B., et al., Effect of luteinizing hormone-releasing hormone agonist on ovarian function after modern adjuvant breast cancer chemotherapy: the GBG 37 ZORO study. Journal of clinical oncology, 2011. 29(17): p. 2334-2341.

22. Gertsch, J., R.G. Pertwee, and V. Di Marzo, Phytocannabinoids beyond the Cannabis plant-do they exist? British journal of pharmacology, 2010. 160(3): p. 523-529.

23. Howlett, A., et al., International Union of Pharmacology. XXVII. Classification of cannabinoid receptors. Pharmacological reviews, 2002. 54(2): p. 161-202.

24. Piomelli, D., The molecular logic of endocannabinoid signalling. Nature Reviews Neuroscience, 2003. 4(11): p. 873-884.

25. Morales, P., D.P. Hurst, and P.H. Reggio, Molecular targets of the phytocannabinoids: a complex picture. Phytocannabinoids, 2017: p. 103-131.

26. Di Marzo, V., The endocannabinoid system in obesity and type 2 diabetes. Diabetologia, 2008. 51(8): p. 1356-1367.

27. Compton, D.R., et al., Pharmacological profile of a series of bicyclic cannabinoid analogs: classification as cannabimimetic agents. Journal of Pharmacology and Experimental Therapeutics, 1992. 260(1): p. 201-209.

28. Davis, M.P., Oral nabilone capsules in the treatment of chemotherapy-induced nausea and vomiting and pain. Expert opinion on investigational drugs, 2008. 17(1): p. 85-95.

29. Huffman, J.W., et al., Structure-activity relationships for 1-alkyl-3-(1-naphthoyl) indoles at the cannabinoid CB1 and CB2 receptors: steric and electronic effects of naphthoyl substituents. New highly selective CB2 receptor agonists. Bioorganic \& medicinal chemistry, 2005. 13(1): p. 89-112.

30. Huffman, J.W., et al., Synthesis and pharmacology of 1-deoxy analogs of CP-47,497 and CP-55,940. Bioorganic \& medicinal chemistry, 2008. 16(1): p. 322-335.

31. Thakur, G.A., et al., Methods for the synthesis of cannabinergic ligands, in Marijuana and Cannabinoid Research. 2006, Springer. p. 113-148.

32. Matsuda, L.A., et al., Structure of a cannabinoid receptor and functional expression of the cloned cDNA. Nature, 1990. 346(6284): p. 561-564.

33. Munro, S., K.L. Thomas, and M. Abu-Shaar, Molecular characterization of a peripheral receptor for cannabinoids. Nature, 1993. 365(6441): p. 61-65.

34. Tsou, K., et al., Cannabinoid CB1 receptors are localized primarily on cholecystokinincontaining GABAergic interneurons in the rat hippocampal formation. Neuroscience, 1999. 93(3): p. 969-975.

35. Shire, D., et al., Molecular cloning, expression and function of the murine CB2 peripheral cannabinoid receptor. Biochimica et Biophysica Acta (BBA)-Gene Structure and Expression, 1996. 1307(2): p. 132-136.

36. Galiègue, S., et al., Expression of central and peripheral cannabinoid receptors in human immune tissues and leukocyte subpopulations. European journal of biochemistry, 1995. 232(1): p. 54-61.

37. Holland, M., et al., Cannabinoid CB1 receptors fail to cause relaxation, but couple via $\mathrm{Gi} / \mathrm{Go}$ to the inhibition of adenylyl cyclase in carotid artery smooth muscle. British journal of pharmacology, 1999. 128(3): p. 597-604. 
38. Oesch, S., et al., Cannabinoid receptor 1 is a potential drug target for treatment of translocation-positive rhabdomyosarcoma. Molecular cancer therapeutics, 2009. 8(7): p. $1838-1845$.

39. Guzman, M., Effects on cell viability. Cannabinoids, 2005: p. 627-642.

40. Velasco, G., C. Sánchez, and M. Guzmán, Towards the use of cannabinoids as antitumour agents. Nature Reviews Cancer, 2012. 12(6): p. 436-444.

41. Felder, C.C., et al., Comparison of the pharmacology and signal transduction of the human cannabinoid CB1 and CB2 receptors. Molecular pharmacology, 1995. 48(3): p. 443-450.

42. Twitchell, W., S. Brown, and K. Mackie, Cannabinoids inhibit $N$-and P/Q-type calcium channels in cultured rat hippocampal neurons. Journal of neurophysiology, 1997. 78(1): p. 43-50.

43. Henry, D.J. and C. Chavkin, Activation of inwardly rectifying potassium channels (GIRK1) by co-expressed rat brain cannabinoid receptors in Xenopus oocytes. Neuroscience letters, 1995. 186(2-3): p. 91-94.

44. Qamri, Z., et al., Synthetic cannabinoid receptor agonists inhibit tumor growth and metastasis of breast cancer. Molecular cancer therapeutics, 2009. 8(11): p. 3117-3129.

45. Caffarel, M.M., et al., 49-tetrahydrocannabinol inhibits cell cycle progression in human breast cancer cells through Cdc2 regulation. Cancer research, 2006. 66(13): p. 6615-6621.

46. Ligresti, A., et al., New potent and selective inhibitors of anandamide reuptake with antispastic activity in a mouse model of multiple sclerosis. British journal of pharmacology, 2006. 147(1): p. 83-91.

47. Ligresti, A., et al., Antitumor activity of plant cannabinoids with emphasis on the effect of cannabidiol on human breast carcinoma. Journal of Pharmacology and Experimental Therapeutics, 2006. 318(3): p. 1375-1387.

48. Galve-Roperh, I., et al., Mechanism of extracellular signal-regulated kinase activation by the CB1 cannabinoid receptor. Molecular pharmacology, 2002. 62(6): p. 1385-1392.

49. Sarfaraz, S., et al., Cannabinoid receptor agonist-induced apoptosis of human prostate cancer cells LNCaP proceeds through sustained activation of ERK1/2 leading to G1 cell cycle arrest. Journal of Biological Chemistry, 2006. 281(51): p. 39480-39491.

50. Mukhtar, H., F. Afaq, and S. Sarfaraz, Cannabinoid Receptors: A Novel Target for Therapy for Prostate Cancer. 2008, WISCONSIN UNIV-MADISON.

51. Preet, A., et al., Cannabinoid receptors, CB1 and CB2, as novel targets for inhibition of non-small cell lung cancer growth and metastasis. Cancer prevention research, 2011. 4(1): p. 65-75.

52. Olea-Herrero, N., et al., The cannabinoid JWH-015 activates $N F \kappa B$ in prostate cancer PC-3 cells: Involvement of CB2 and PI3K/Akt. F1000Research, 2011. 2.

53. Kitatani, K., J. Idkowiak-Baldys, and Y.A. Hannun, The sphingolipid salvage pathway in ceramide metabolism and signaling. Cellular signalling, 2008. 20(6): p. 1010-1018.

54. Velasco, G., et al., Cannabinoids and ceramide: two lipids acting hand-by-hand. Life sciences, 2005. 77(14): p. 1723-1731.

55. Guzmán, M., C. Sánchez, and I. Galve-Roperh, Control of the cell survival/death decision by cannabinoids. Journal of molecular medicine, 2001. 78(11): p. 613-625.

56. Carracedo, A., et al., Cannabinoids induce apoptosis of pancreatic tumor cells via endoplasmic reticulum stress-related genes. Cancer research, 2006. 66(13): p. 67486755.

57. Bifulco, M., et al., Control by the endogenous cannabinoid system of ras oncogenedependent tumor growth. The FASEB Journal, 2001. 15(14): p. 1-17. 
58. Park, J.M., et al., Antiproliferative mechanism of a cannabinoid agonist by cell cycle arrest in human gastric cancer cells. Journal of cellular biochemistry, 2011. 112(4): p. 1192-1205.

59. McCubrey, J.A., et al., Roles of the Raf/MEK/ERK pathway in cell growth, malignant transformation and drug resistance. Biochimica et Biophysica Acta (BBA)-Molecular Cell Research, 2007. 1773(8): p. 1263-1284.

60. Okano, J.-i. and A.K. Rustgi, Paclitaxel induces prolonged activation of the Ras/MEK/ERK pathway independently of activating the programmed cell death machinery. Journal of Biological Chemistry, 2001. 276(22): p. 19555-19564.

61. Portella, G., et al., Inhibitory effects of cannabinoid CB1 receptor stimulation on tumor growth and metastatic spreading: actions on signals involved in angiogenesis and metastasis. The FASEB Journal, 2003. 17(12): p. 1771-1773.

62. Casanova, M.L., et al., Inhibition of skin tumor growth and angiogenesis in vivo by activation of cannabinoid receptors. The Journal of clinical investigation, 2003. 111(1): p. 43-50.

63. Fan, F., et al., Cannabinoid receptor down-regulation without alteration of the inhibitory effect of CP 55,940 on adenylyl cyclase in the cerebellum of CP 55,940tolerant mice. Brain research, 1996. 706(1): p. 13-20.

64. Nong, L., et al., Altered cannabinoid receptor $m R N A$ expression in peripheral blood mononuclear cells from marijuana smokers. Journal of Neuroimmunology, 2002. 127(1-2): p. 169-176.

65. Martini, L., et al., Ligand-induced down-regulation of the cannabinoid 1 receptor is mediated by the G-protein-coupled receptor-associated sorting protein GASP1. The FASEB Journal, 2007. 21(3): p. 802-811.

66. Romero, J., et al., Time-course of the cannabinoid receptor down-regulation in the adult rat brain caused by repeated exposure to 49-tetrahydrocannabinol. Synapse, 1998. 30(3): p. 298-308.

67. Leterrier, C., et al., Constitutive endocytic cycle of the CB1 cannabinoid receptor. Journal of biological chemistry, 2004. 279(34): p. 36013-36021.

68. Wu, D.F., et al., Role of receptor internalization in the agonist-induced desensitization of cannabinoid type 1 receptors. Journal of neurochemistry, 2008. 104(4): p. 11321143.

69. Rozenfeld, R., Type I cannabinoid receptor trafficking: all roads lead to lysosome. Traffic, 2011. 12(1): p. 12-18.

70. Grimsey, N.L., et al., Cannabinoid receptor 1 trafficking and the role of the intracellular pool: implications for therapeutics. Biochemical pharmacology, 2010. 80(7): p. 1050-1062.

71. Grimsey, N.L., et al., Cannabinoid receptor 2 undergoes Rab5-mediated internalization and recycles via a Rab11-dependent pathway. Biochimica et Biophysica Acta (BBA)-Molecular Cell Research, 2011. 1813(8): p. 1554-1560.

72. Tappe-Theodor, A., et al., A molecular basis of analgesic tolerance to cannabinoids. Journal of Neuroscience, 2007. 27(15): p. 4165-4177.

73. Biswas, D.K., et al., Epidermal growth factor-induced nuclear factor $\kappa B$ activation: $a$ major pathway of cell-cycle progression in estrogen-receptor negative breast cancer cells. Proceedings of the National Academy of Sciences, 2000. 97(15): p. 8542-8547.

74. Roch, F., G. Jiménez, and J. Casanova, EGFR signalling inhibits Capicua-dependent repression during specification of Drosophila wing veins. 2002.

75. Gustafsson, K., et al., Cannabinoid receptor-mediated apoptosis induced by $R(+)$ methanandamide and Win55, 212-2 is associated with ceramide accumulation and p38 
activation in mantle cell lymphoma. Molecular pharmacology, 2006. 70(5): p. 16121620.

76. Derkinderen, P., et al., Cannabinoids activate p38 mitogen-activated protein kinases through CB1 receptors in hippocampus. Journal of neurochemistry, 2001. 77(3): p. 957-960.

77. GÓMEZ DEL PULGAR, T., G. Velasco, and M. Guzman, The CB1 cannabinoid receptor is coupled to the activation of protein kinase B/Akt. Biochemical Journal, 2000. 347(2): p. 369-373.

78. Yamboliev, I.A., et al., Evidence for modulation of smooth muscle force by the p38 MAP kinase/HSP27 pathway. American Journal of Physiology-Heart and Circulatory Physiology, 2000. 278(6): p. H1899-H1907.

79. Cai, T., et al., Manganese induces the overexpression of $\alpha$-synuclein in PC12 cells via ERK activation. Brain research, 2010. 1359: p. 201-207.

80. Kralova, J., et al., p38 MAPK plays an essential role in apoptosis induced by photoactivation of a novel ethylene glycol porphyrin derivative. Oncogene, 2008. 27(21): p. 3010-3020.

81. Guzman, M., Cannabinoids: potential anticancer agents. Nature reviews cancer, 2003. 3(10): p. 745-755.

82. Kolesnick, R.N. and M. Krönke, Regulation of ceramide production and apoptosis. Annual review of physiology, 1998. 60(1): p. 643-665.

83. Galve-Roperh, I., et al., Anti-tumoral action of cannabinoids: involvement of sustained ceramide accumulation and extracellular signal-regulated kinase activation. Nature medicine, 2000. 6(3): p. 313-319.

84. Li, N. and M. Karin, [25] Signaling pathways leading to nuclear factor- $\kappa B$ activation. Methods in enzymology, 2000. 319: p. 273-279.

85. Frelin, C., et al., Targeting NF- $\kappa B$ activation via pharmacologic inhibition of IKK2induced apoptosis of human acute myeloid leukemia cells. Blood, 2005. 105(2): p. 804811.

86. Yamamoto, Y. and R.B. Gaynor, Role of the NF-kB pathway in the pathogenesis of human disease states. Current molecular medicine, 2001. 1(3): p. 287-296.

87. Sancho, R.o., et al., Anandamide inhibits nuclear factor- $\kappa B$ activation through a cannabinoid receptor-independent pathway. Molecular pharmacology, 2003. 63(2): p. 429-438.

88. Dolcet, X., et al., NF- $k B$ in development and progression of human cancer. Virchows archiv, 2005. 446(5): p. 475-482.

89. Ellert-Miklaszewska, A., B. Kaminska, and L. Konarska, Cannabinoids down-regulate PI3K/Akt and Erk signalling pathways and activate proapoptotic function of Bad protein. Cellular signalling, 2005. 17(1): p. 25-37.

90. Hart, S., O.M. Fischer, and A. Ullrich, Cannabinoids induce cancer cell proliferation via tumor necrosis factor $\alpha$-converting enzyme (TACE/ADAM17)-mediated transactivation of the epidermal growth factor receptor. Cancer research, 2004. 64(6): p. 1943-1950.

91. Chan, G.C.-K., Hinds, T.R., Impey, S., Storm, D.R., Hippocampal neurotoxicity of 49tetrahydrocannabinol. Journal of Neuroscience, 1998. 18(14): p. 5322-5332.

92. Abood, M.E., Rizvi, G., Sallapudi, N., McAllister, S.D., Activation of the CB1 cannabinoid receptor protects cultured mouse spinal neurons against excitotoxicity. Neuroscience Letters, 2001. 309(3): p. 197-201.

93. Stefan Hart, O.M.F., Axel Ullrich, Cannabinoids Induce Cancer Cell Proliferation via Tumor Necrosis Factor $\alpha$-Converting Enzyme (TACE/ADAM17)-Mediated 
Transactivation of the Epidermal Growth Factor Receptor. Cancer Research, 2004. 64(6): p. 1943-1950.

94. Brenneisen R, E.A., Elsohly MA, Henn V, Spiess Y., The effect of orally and rectally administered delta 9-tetrahydrocannabinol on spasticity: a pilot study with 2 patients. Int J Clin Pharmacol Ther, 1996. 34(10): p. 446-52.

95. Heishman SJ, H.M., Henningfield JE, Cone EJ., Acute and residual effects of marijuana: profiles of plasma THC levels, physiological, subjective, and performance measures. Pharmacol Biochem Behav, 1990. 37(3): p. 561-5.

96. Maria G. Sanchez, L.R.-L., Ana M. Sánchez, Inés Díaz-Laviada, Activation of phosphoinositide 3-kinase/PKB pathway by $C B 1$ and $C B 2$ cannabinoid receptors expressed in prostate $P C-3$ cells. Involvement in Raf-1 stimulation and NGF induction. Cellular Signalling, 2003. 15(9): p. 851-859.

97. Huffman, J.W., CB2 Receptor Ligands. Mini Reviews in Medicinal Chemistry, 2005. 5(7): p. 641-649.

98. Marriott, K.S. and J.W. Huffman, Recent advances in the development of selective ligands for the cannabinoid CB(2) receptor. Curr Top Med Chem, 2008. 8(3): p. 187204.

99. Romon, R., et al., Nerve growth factor promotes breast cancer angiogenesis by activating multiple pathways. Molecular Cancer, 2010. 9(1): p. 157.

100. Reichardt, L.F., Neurotrophin-regulated signalling pathways. Philosophical Transactions of the Royal Society B: Biological Sciences, 2006. 361(1473): p. 15451564.

101. Annette K Larsen, D.O., Karima El Ouadrani, Amélie Petitpre, Targeting EGFR and $\operatorname{VEGF}(R)$ pathway cross-talk in tumor survival and angiogenesis. Pharmacol Ther, 2011. 131(1): p. 80-90.

102. Baselga, The EGFR as a target for anticancer therapy-focus on cetuximab. European Journal of Cancer, 2001. 37, Supplement 4(0): p. 16-22. 